\title{
Globally Linear Embedding of Biometric Scores: An Empirical Study
}

\author{
Sudeep Sarkar and Pranab Mohanty
}

\begin{abstract}
We consider if it is possible to embed biometric similarity scores in low-dimensional, inner product spaces. Starting from a matrix of similarity scores between target and query templates, as computed by a biometric algorithm, we seek to represent the templates as points in a low-dimensional space such that their inner products approximates the neighborhood relationship in the given similarity matrix. The process involves the minimization of the Frobenius norm between the similarities, transformed by a monotonic function, and the inner product of the embedding coordinates. Possible space of monotonic functions are those that transform the statistics of the given scores into a Gumbel distribution, a limiting case extreme-value distribution. The minimization is then over the space of Gumbel parameters and the coordinates. We solve this in a two-step iterative process. Given the Gumbel parameters, we find the coordinates using the Singular Value Decomposition (SVD) of the transformed similarities. The vectors of the decomposition gives us the coordinates. We search for the Gumbel parameters using simplex search, optimizing a rank dispersion index that captures the average fall in rank of the top $k$-ranked queries for each target, based on the estimated coordinates.
\end{abstract}

We experiment with four different biometrics, namely, gait, face, fingerprint, and voice. For each biometric, we have two different similarity matrices, either from two different algorithms on the same dataset or using the same algorithm on two different datasets. For gait, face, and fingerprint, we used similarities computed on standard datasets, i.e., the HumanID Gait Challenge, FERET dataset, and NIST Biometrics Score Set, respectively. For voice, we used our own data, collected indoor and outdoors. The similarity matrix sizes were 122 by 958,1196 by 956,168 by 252,500 by 1000 , for the four modalities. We find that rank dispersion can be kept small $(<$ $1 \%$ of the target set size) for a low-dimensional approximation that is $20 \%$ of the maximum dimensions needed for perfect embedding. We also find that the quality of the embedding is the best for face, followed by gait, fingerprint, and then voice.

\section{INTRODUCTION}

Biometric scores are not random. There is structure in the scores. One way to get at this structure is through statistical analysis. This has been the dominant mode of analysis. There are studies that statistically model similarity scores to predict the performance of the algorithm on large data sets based on results on small data sets [1], [2], [3]. For instance, Grother and Phillips [4] proposed a joint density function to predict match (genuine) scores and non-match (imposter) scores. Apart from face-based biometrics, methods have been also proposed to model and predict performances for other biometric modalities [5]. There are also investigations that

S. Sarkar is with Faculty of Computer Science and Engineering, University of South Florida, 4202 E Fowler Ave., ENB 118, Tampa, Florida 33620 sarkar@cse.usf.edu

Pranab Mohanty was with University of South Florida and is now at Aware Inc. pkmohant@cse.usf .edu study the statistics of scores to design better biometric fusion algorithms [6]. However, unlike these approaches, we seek to unravel the geometric structure that is implicitly present among the similarity scores.

Given a distance (or similarity) matrix, there is a rich body of work in multidimensional scaling (MDS) [7] that can be used to find low-dimensional coordinates for each point such as that distances between these points match the given distance matrix, under an appropriate error norm. Typically, these methods start from a square distance matrix between every pair of objects. Most recent methods consider nonlinear methods to arrive at the most parsimonious space (manifold) [8], [9] that can be used to place the points. Such methods have been used to visualize mostly (in 2 or 3 dimensions) the underlying manifold of face recognition algorithms [10]. In this paper, we empirically study if it is possible to embedded biometric similarity matrices for a variety of modalities in a globally linear space. The stress on linearity is not just based on appeal to Occam's razor, but also because of possible impact on template security, privacy, indexing. In [11] we have shown that if we can build globally linear models of the distances, then it is possible to construct approximating linear models that map biometric templates to distances, as computed by a recognition algorithm. Based on this linear model, it is possible to reconstruct any target template given just the similarity scores of this target template with a set of break-in query templates. We had demonstrated this for faces. In [12] we have also shown how a linear model can be used to construct algorithm-specific indexing scheme for faces, with 20 times indexing speedups.

We start by formulating the theory behind the particular version of the embedding problem that is relevant to us in the biometrics context. We typically have a rectangular matrix of similarities (or dissimilarities) between target and query sets. These sets do not overlap in terms of the exact templates, but they can have intersection in terms of the subjects. One property of biometric similarity scores is that if we remap or transform these scores using a monotonic transform the biometric performance does not change. We exploit this to arrive at a compact representation of the underlying space. We design the monotonic transformation based on extreme value distributions since decisions in biometric identification are essentially with extreme values, i.e. minimum (or maximum) scores. Technically, we have a non-metric, inner product based embedding problem, which we solve in a minimization framework. The formulation can handle both similarities and dissimilarity scores. Perhaps the closest related works in terms of the underlying theory are those that use multidimensional scaling (MDS) to derive 
models for standard classifiers such as nearest neighborhood, linear discriminant analysis, and linear programming problem from the dissimilarity scores between objects [13]. Roth et al. [14] also suggest a similar framework, where pairwise distance information is embedded in the Euclidean space, and equivalence is drawn between several clustering approaches with similar distance based learning approaches.

In the experimental part, we consider four, diverse biometrics, namely, face, gait, fingerprint, and voice and investigate if their similarity matrices can be embedded in linear, low dimension spaces. For each biometric, we consider two different similarity matrices, either produced using two different algorithms on the same dataset or produced by applying the same algorithm on two different datasets. We ground our conclusions based on experiments conducted in a train and test framework.

\section{THEORY}

Let $\mathbf{S}$ be a $N_{t}$ by $N_{q}$ sized matrix of similarities between target and query sets. The scalar $s_{t q}$ is the similarity between the $t$-th target template with the $q$-th query template, as computed by a biometric algorithm. We express our theory in terms of similarity matrices, but it also holds for dissimilarity matrices. We just turn them into similarities by considering difference with some fixed value. We do not have access to the actual templates, nor do we need them in this study. There is no constraint on the composition of the targets and queries, except that the query set include templates from a subset of subjects in the target. Expressed in another way, the matrix $\mathbf{S}$ should include match and non-match scores. Note that we are insisting on the overlap of subjects between the query and target sets, not the actual templates. As a consequence of this, the matrix $\mathbf{S}$ is not symmetric, nor do we have ones (or maximum possible similarity value) along the diagonal.

To shed light on the geometric structure underlying the similarity matrix, we would like to represent each target and query template as points in a low-dimensional spaces such that the inner product (dot-product) between the coordinates captures the ordering information that is in S. For biometrics, the absolute values of the similarities are not important. Monotonic transformation of similarities does not affect identification or verification performance. The rank of a target for a query template will not change with such transformations; hence, the identification rate will not change. For the verification scenario, monotonic transformation will just change the value of the decision threshold, not the verification rate for a particular false alarm rate.

We capture this flexibility in the optimization criterion as follows. Let $\mathbf{x}_{t}$ be a $k$-dimensional vector representing the $t$-th target template. Each target is associated with the rows of $\mathbf{S}$. Likewise, $\mathbf{y}_{q}$ is a $k$-dimensional vector representing the $q$-th query template, associated with the columns of $\mathbf{S}$. The inner product between these points, $\mathbf{x}_{t}^{T} \mathbf{y}_{q}$, should be close to a monotonic transformation of the corresponding algorithm computed similarity value, $f\left(s_{t q}\right)$. The coordinates are those that minimize the following optimality criterion.

$$
\min _{\left\{\mathbf{x}_{t}, \mathbf{y}_{q}\right\}} \sqrt{\sum_{t} \sum_{q}\left(\mathbf{x}_{t}^{T} \mathbf{y}_{q}-f\left(s_{t q}\right)\right)^{2}}
$$

or equivalently in matrix notation

$$
\min _{\{\mathbf{X}, \mathbf{Y}\}} \sqrt{\left.\left(\mathbf{X}^{T} \mathbf{Y}-\mathbf{S}_{f}\right)\right)^{2}}
$$

where $\mathbf{X}=\left[\mathbf{x}_{1}, \cdots, \mathbf{x}_{N_{t}}\right], \mathbf{y}=\left[\mathbf{y}_{1}, \cdots, \mathbf{y}_{N_{q}}\right]$, and $\mathbf{S}_{f}$ is a matrix, $\mathbf{S}$, with entries transformed by the monotonic function $f($.$) .$

\section{A. Inner-product Embedding}

Given a function $f($.$) , whose choice we discuss in the$ next section, Eq. 2 can be minimized by choosing $\mathbf{X}$ and $\mathbf{Y}$ based on the singular valued decomposition of $\mathbf{S}_{f}=\mathbf{U} \Sigma \mathbf{V}^{T}$, where $\mathbf{U}$ and $\mathbf{V}$ are the matrices with orthonormal columns and $\Sigma$ is a diagonal matrix with singular values.

$$
\mathbf{X}=\Sigma^{0.5} \mathbf{U}^{T} \quad \text { and } \quad \mathbf{Y}=\Sigma^{0.5} \mathbf{V}^{T}
$$

In general, the dimension of $\mathbf{X}$ will be $N_{t}$ by $N_{t}$, giving us $N_{t}$ dimensional, perfect embedding of the similarities. This is not particularly interesting. Any $m$ by $n$ matrix can be embedded in $\min (m, n)$ dimensional space. We exploit the unique property of SVD decompositions that keeping the top$k$ singular values in $\Sigma$ and setting the rest to zero will result in the optimal (matrix) rank $K$ minimization of Eq. 2. Thus, we consider only the $K$ columns of the orthogonal vectors in SVD to construct the $K$-dimensional approximating space.

$\mathbf{X}_{K}=\sqrt{\Sigma_{K}}\left[\mathbf{u}_{1}, \cdots, \mathbf{u}_{K}\right]^{T}$ and $\mathbf{Y}_{K}=\sqrt{\Sigma_{K}}\left[\mathbf{v}_{1}, \cdots, \mathbf{v}_{K}\right]^{T}$

The columns of $\mathbf{X}_{K}$ and $\mathbf{Y}_{K}$ gives us the coordinates of the target (rows) and query (column) templates in a $K$ dimensional space. This collection of points, along with their inner product, makes explicit the geometry underlying the similarity matrix. This placement of the points will shift depending on the choice of the transformation, $f($.$) . We would$ like to choose $f($.$) such that nearest neighbor relationship is$ preserved to the largest extent. This we discuss, next.

\section{B. Gumbel Transformation}

Among possible choices of the monotonic transformation, we are most interested in the ones that capture the statistics of the low-ranked scores. In biometrics context, it is important to capture the structure among the larger scores, which express most similarity to the target templates. In other words, the extreme values of the similarity score distributions are of most interest. The target-query similarity computation process typically involves a maximization process over parameters such as geometric alignment, illumination, etc. The maximum value of the similarity under these nuisance variations are chosen to characterize the match between two templates. Note that in case where registration information, such as eye location for faces, is provided manually or computed automatically, we can still look at the overall process 


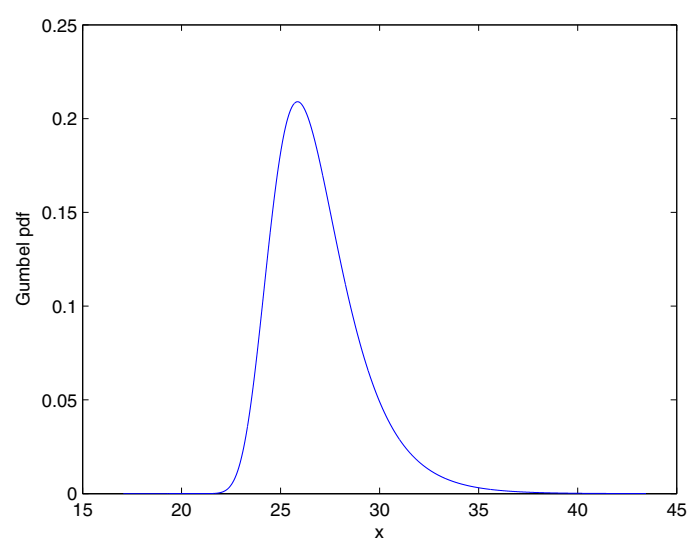

Fig. 1. An example of the Gumbel probability density function (pdf) for the maximum value. This particular example was estimated for transforming one of the fingerprint similarity matrices used in this study.

as an optimization problem than has been broken down into two parts. Choosing values other than the specified registration values will result in lower similarities. Thus, extreme (maximum) value distributions are appropriate for similarity scores. Analogously, minimum extreme value distributions would be appropriate for dissimilarities. More specifically, we design the function, $f($.$) such that the distribution of$ $f($.$) represents the distribution of extreme values.$

From extreme valued statistics [15] we know that for samples taken from a well behaving but arbitrary distribution, the distribution of the extreme values (minimum or maximum of a set of $n$ variables), as $n \rightarrow \infty$, converges to one of the three types of extreme value distributions. The choice between the three forms depend on the domain of the variables. This is like the central limit theorem, but for extreme values. For unbounded domain of the scores, the Gumbel distribution is appropriate. Its cumulative distribution function (cdf) is given by:

$$
F(s ; \alpha, \beta)=\exp -\left[\exp -\left(\frac{s-\alpha}{\beta}\right)\right]
$$

where $\alpha$ and $\beta$ are the mode and the scale parameters of the distribution. A form of the Gumbel probability density function (pdf), actually derived for one of the fingerprint similarity matrices, is shown in Fig. 1. Note the heavy tailed nature of the distribution towards large values. To derive the monotonic mapping function, we will work with cdf instead of the pdf. Let the cdf for the similarity scores, as estimated form the whole matrix, be denoted by $G(s)$. The required transformation to turn the scores into extreme value distributions is given by

$$
f\left(s_{t q}\right)=F^{-1}\left(G\left(s_{t q}\right) ; \alpha, \beta\right)
$$

This transformation is monotonic because the underlying cdf functions are, by definition, monotonic. As we vary the parameters $\alpha, \beta$ the approximating geometry, captured by the estimated coordinates (Eq. 4), will change. We choose the parameters so that the local dispersion index (LDI), a measure that we define later in this paper, is optimized. The local dispersion index measure captures the fall in rank (or dispersion) of the $k$-nearest target neighbors for each query, as computed based on the given similarities, with those computed using the approximating inner product distance.

$$
\left\{\alpha^{*}, \beta^{*}\right\}=\arg \min _{\alpha, \beta} \operatorname{LDI}_{k}\left(\mathbf{S}, \mathbf{X}_{K}^{T} \mathbf{Y}_{K}\right)
$$

\section{Local Dispersion Index}

This is a measure of how the local neighborhood around a template point is different between the original space and the approximating linear space. One way to capture this is using a rank based measures. If we consider the nearest neighbor to a target template, with respect to the original similarity scores, what is the expected rank of it in the approximating linear space? Generalizing this question, if we consider the $k$-nearest neighbors to a target template, with respect to the original similarity scores, what is the expected highest rank of this set of neighbors in the approximating linear space? In other words, how are the $k$-nearest neighbors dispersed in the linear space? If the answer is $k$, then there is no distortion in the local neighborhood upto rank $k$. Of course, for real data, it will be higher than $k$.

We compute this local dispersion measure as follows. We have two similarity matrices, the original matrix $\mathbf{S}$ and approximating matrix $\mathbf{R}$, both over the same target and query sets, in the same order, i.e. corresponding entries are for the same pair of target and query comparisons. For each query, we rank the target images based each of the similarity matrices and then consider the identities of the $k$ nearest neighbors, based on the original similarity matrix. Let $\mathbf{i}_{q}=\arg \operatorname{sort}\left(s_{1 q}, \cdots, s_{N_{t} q}\right)$ represent the permuted indices representing the sorted order of the $q$-th column in the matrix S. Similarly $\mathbf{j}_{q}=\arg \operatorname{sort}\left(r_{1 q}, \cdots, r_{N_{t} q}\right)$ represent the permuted indices representing the sorted order of the $q$ th column in the approximating matrix $\mathbf{R}$. Using these, we define the local manifold agreement at rank $k$ to be

$$
\operatorname{LDI}_{k}(\mathbf{S}, \mathbf{R})=\frac{1}{N_{t}} \frac{1}{N_{q}} \sum_{q} \operatorname{Dispersion}(q)
$$

where

$$
\begin{gathered}
\operatorname{Dispersion}(q)= \\
\arg \min _{m}\left(\left\{i_{q}(1), \cdots, i_{q}(k)\right\} \in\left\{j_{q}(1), \cdots, j_{q}(m)\right\}\right)-k
\end{gathered}
$$

$\operatorname{Dispersion}(q)$ measures the rank dispersion of the $q$-th query template. It is zero if the top $k$ target templates based on the original similarity matrix are the same as those based on the approximating scores. The value increases as the top $k$ rank entries are dispersed down the ranks. Note that we do not consider if the $k$ neighbors are correct or wrong matches. This makes it a stronger measure than rank-based, identification rates in Cumulative Match Curves (CMCs). Two algorithms can have the same rank $k$ identification but the $\mathrm{LDI}_{1}$ can be lower for one. For the LDI to be high, the identities of both the correct and incorrect matches should agree. Since we want to capture the underlying geometry of the scores, both matches and non-matches are important. 
Also, note that $\mathrm{LDI}_{k}$ is normalized by the size of the target set, so that we can compare across similarity matrices of different sizes.

This type of measure is useful in giving us some indication of the usefulness of the linear embedding for indexing. The local dispersion index will give us an idea of how many neighbors to consider in this linear space, given a query template so that one we are likely to find all the $k$ nearest neighbors as computed by the underlying biometric algorithm. As an example interpretation, an $\mathrm{LDI}_{1}=0.01$ means that the nearest neighbor of any query, based on the original similarity matrix, is expected to be contained in the nearest $1 \%$ of the target set size neighbors in the approximating space. This translates to a 100-fold saving when indexing just the nearest neighbor.

\section{Projection of New Sample}

Let us say we have learned a low-dimensional space that approximates a given similarity matrix fairly well, how can we find the coordinates of a new query, $\mathbf{y}_{n}$, in this space. We will assume that we have the similarities of this query with the target set, $\mathbf{s}_{n}=\left\{s_{1 n}, \cdots, s_{t n}, \cdots, s_{N_{t} n}\right\}$, as computed by the underlying algorithm. The differences of these similarities with the dot product of the new query coordinates and the target coordinates, given by $\mathbf{X}$, should be minimized.

$$
\min _{\mathbf{y}_{n}}\left\|\mathbf{X}^{T} \mathbf{y}_{n}-\mathbf{s}_{n}\right\|
$$

The above least square solution can be expressed as

$$
\mathbf{y}_{n}=\left(\mathbf{X X}^{T}\right)^{-1} \mathbf{X} \mathbf{s}_{n}
$$

Using the expression of the coordinates in Eq. 4, we can simplify the expression to

$$
\mathbf{y}_{n}=\left(\Sigma_{K}\right)^{-\frac{1}{2}} \mathbf{U}^{T} \mathbf{s}_{n}
$$

\section{CHOICE OF THE BIOMETRICS}

We report our investigations of four diverse biometrics, face, gait, fingerprint, and voice. For face biometrics we chose performance on the FERET dataset [16], specifically the fa-fb and dup-1 experiments. For gait, we chose the HumanID Gait Challenge dataset [17]. We used fingerprint scores from the NIST [18]. For voice, we used an inhouse collection of voice samples. For speech samples, each user utters a fixed phrase, "University of South Florida 33620". The indoor and outdoor samples were captured using regular cardioid microphones. The sampling frequency was $11 \mathrm{kHz}$. For this study, only samples using the cardioid microphone were used to avoid effects of equipment bias in the indoor/outdoor scenario. The outdoor environment was near the entrance to a building where the hypothetical authentication would take place. The indoor environment was similar to a quiet office environment. Table I lists the similarity matrix sizes and the number of subjects in captured in each matrix.

For each modality, we considered two different similarity matrices, corresponding either to the same underlying algorithm on different datasets, or to different algorithms
TABLE I

BREAKDOWN OF SIMILARITY MATRICES SIZES USED IN THIS STUDY.

\begin{tabular}{ccc}
\hline Biometric & Source & Matrix Size \\
\hline \hline Gait & Gait Challenge & 122 by 958 \\
\hline Face & FERET & 1196 by 1917 \\
\hline Voice & USF & 168 by 252 \\
\hline Fingerprint & NIST & 500 by 1000 \\
\hline
\end{tabular}

on different datasets. We had to consider two instances in this mixed manner due to the constraints imposed by the availability of datasets and recognition algorithms. For gait, we chose two top performing gait recognition algorithms and their performance on the HumanID gait challenge dataset. For face, we chose the Elastic Bunch Graph Matching (EBGM), as implemented by the CSU group [19], and a commercial algorithm from 2002. For fingerprint, we used the left-index and the right-index similarity scores in the NIST dataset. For voice, the recognition algorithm was based on dynamic time warping but it was run on two different sets of templates.

\section{RESULTS}

We start by discussing results on the entire similarity matrices. How well are the local manifold structure captured by the global approximation that we have computed for each biometric? Fig. 2 (a) and (b) shows the local dispersion indices at ranks one and five, respectively, for two instances for each of the biometrics, based on the entire similarity matrix. The dispersion index depends on the dimensionality of the approximating space. In the limit, any $N$ data points can be perfectly embedded in $N-1$ dimensions. Errors will arise as we reduce the approximating dimensions. To normalize this dimensional measure across the datasets, all of which have different sizes, we consider the ratio of the reduced dimension to the dimension needed for perfect embedding, i.e. total number of target and query templates. This ratio is plotted along the horizontal axis. We find that the rank dispersion can be kept small $(<1 \%$ of the target set size) for a low-dimensional approximation that is $20 \%$ of the maximum dimensions needed for perfect embedding. We also find that the quality of the embedding is the best for face, followed by gait, fingerprint, and then voice. This conclusion holds quite well when we consider the agreement at rank five too.

\section{A. Train and Test}

To judge the ability to generalize the observed properties, we conducted a train and test study. The approximation was learnt on one matrix and tested on the other. Because of the diverse nature of the available similarities, we had to adopt different strategies to construct the train and test sets for each modality. For gait, we separated the query (columns) into two sets. The train set was the similarity of the target (row) templates with one query subset and the test set were the similarities of the target with the other query subset. For face, we picked the fa-fb set for training and dup-1 


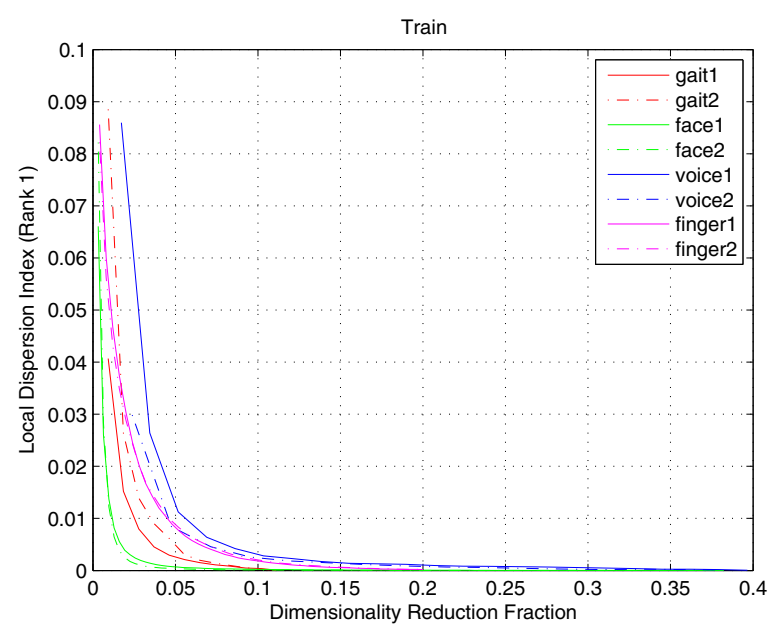

(a)

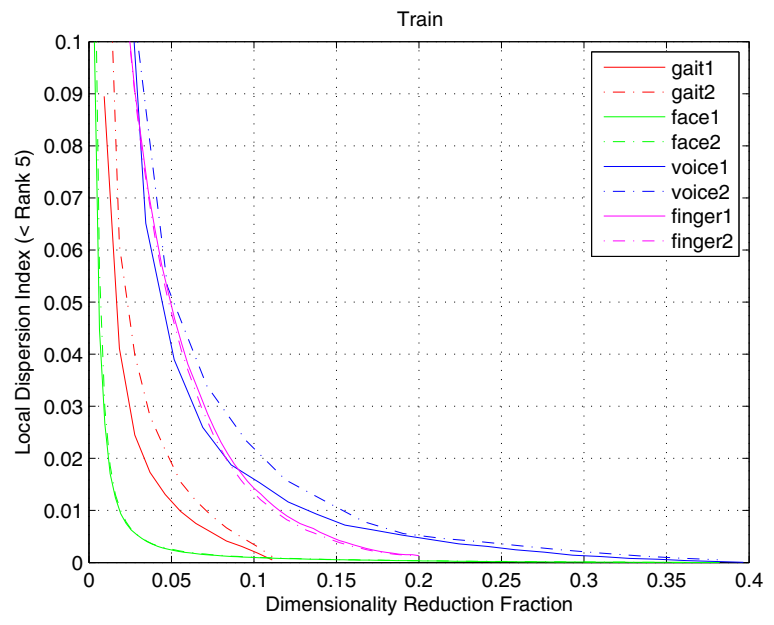

(b)

Fig. 2. Local dispersion index versus the size of the reduced dimensional space, expressed as fraction of the size needed for perfect fit. (a) and (b) shows the (train) set performance for the entire similarity matrices at ranks one and five, respectively.

set for testing. Both the sets are for the same target set but different queries. For voice, we separated the queries into two subsets, one for training and the other for testing. For the NIST fingerprint data we considered a separate query set for testing, but the same target set for training and testing. The fingerprint dataset contained one match score per target, which meant that the testing matrix did not contain any match scores. The only match score per target was included in the training step. To compute the coordinates for the test matrices, we used the new sample projection form in Eq. 12. Fig. 3 shows the test performances at ranks one and five. We see that under test conditions the earlier conclusions about face and gait hold up. For fingerpint, the results are particularly interesting since the testing set did not include any match scores, demonstrating that the manifold captures the geometry among non-match scores fairly well. For voice, we do have lower dispersions but with a slightly larger dimension.

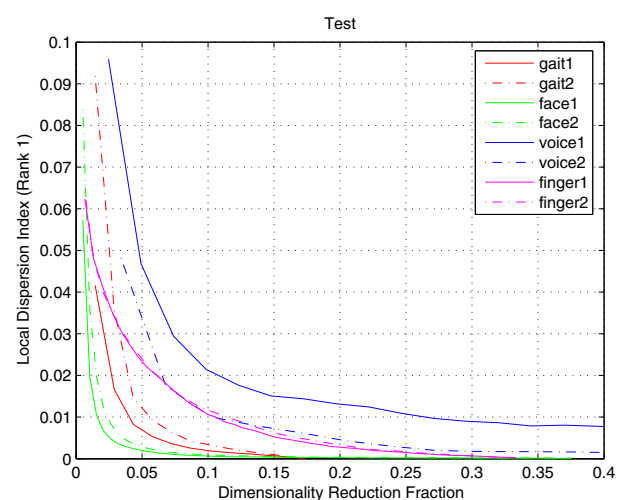

(a)

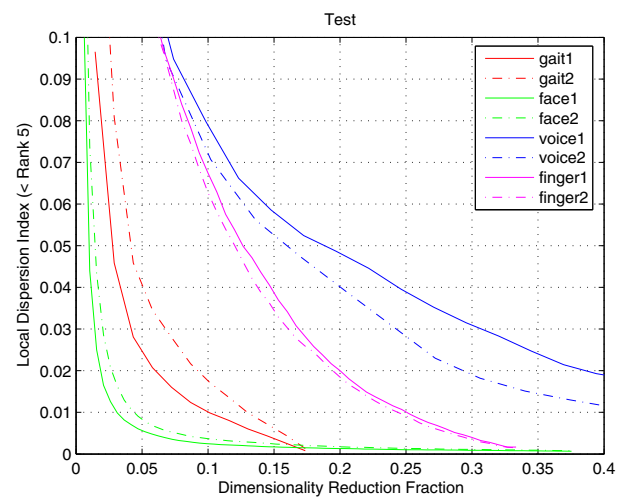

(b)

Fig. 3. Local dispersion index versus the size of the reduced dimensional space, expressed as fraction of the size needed for perfect fit. (a) and (b) shows the test set performance at ranks one and five, respectively.

\section{Monotonic Mapping Function}

How different were the monotonic mapping functions for the different instances of the biometric similarity matrices? Fig. 4 plots the different, optimized, monotonic mapping functions, registered together and scaled for trend comparison purposes. Each function is actually over different domain and range of values. We notice that the mapping function for the fingerprint scores is markedly different from the others. This is not surprising if we consider the nature of the fingerprint scores. They appear to be quantized values. They also appear to be normalized, although we are not sure about this. None of the mapping functions are linear.

What happens if we do not use any transformations? The performance degrades. Fig. 5 shows a performance point for a reduced dimensional space that is $15 \%$ of the maximum possible for each case. We show the local dispersion index for one similarity matrix per biometric. This selection is just for clarity of presentation. The trends are the same for other matrices as well. We compare the use of Gumbel-based transformation, with no transformation, and with Gaussianbased transformation. Our choice of the Gaussian form as comparison is based on its ubiquitous use and its efficacy in a variety of real world situations. We notice that while the nature of transformation does not have much impact for 


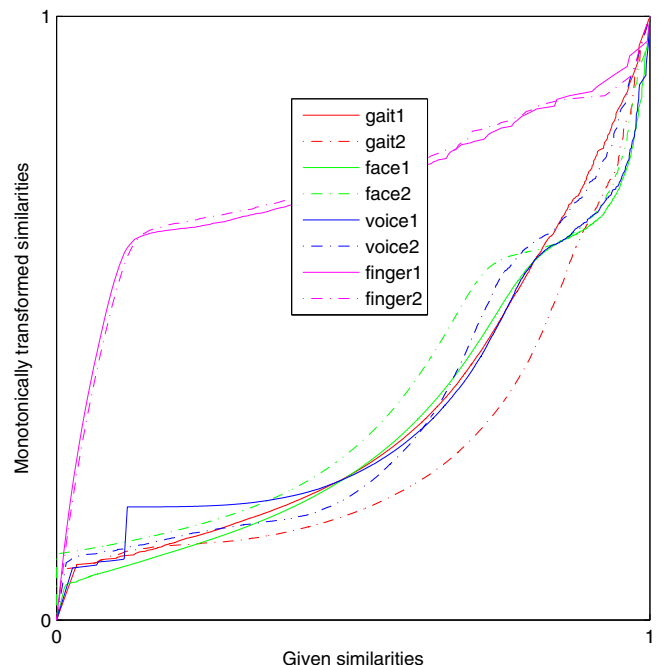

Fig. 4. The optimal similarity remapping function, $f($.$) that was estimated$ for each biometric score set. Each plot has its own range and domain for its mapping. We have scaled them here to the interval $[0,1] \rightarrow[0,1]$ so that we can display them in the same plot.

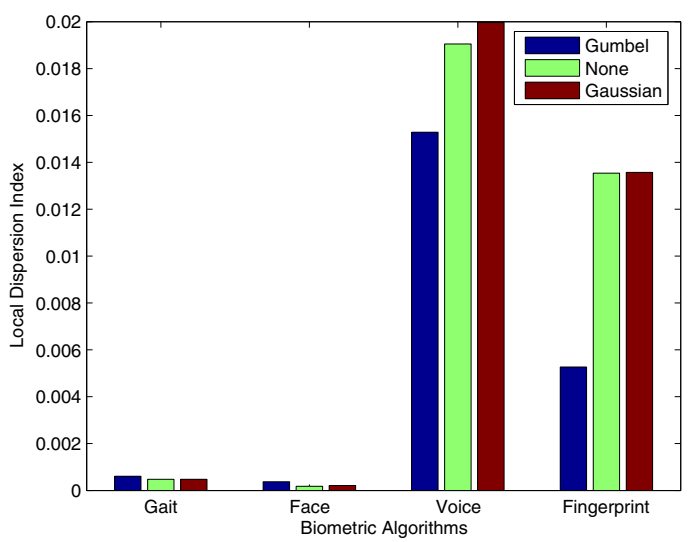

Fig. 5. Comparison of the use of Gumbel-based transformation with Gaussian-based and with no transformation. The dispersion index is shown for a reduced dimensional space that is $15 \%$ of the maximum. For clarity, we show performance only on one dataset per biometric.

face and gait scores used in this study, it does affect voice and fingerprint approximations. There is marked reduction of dispersion with the use of Gumbel-based transformation.

\section{CONCLUSIONS}

Using a variety of biometrics, different algorithms, and well known datasets, we found that biometric similarity matrices allow for good quality low-dimensional embedding in globally linear spaces. We saw that gait and face biometric scores can be approximated fairly well with very low-dimensions. Fingerprint and voice require somewhat higher number of dimensions, but their scores can also be approximated fairly well. Our study strongly suggests that biometric scores should not be just looked upon from a statistical point of view, but that there is a strong underlying, geometric nature. We are excited about the global nature of the approximation. In the short term, this global approximation can be used to reconstruct templates and to build better indexing mechanisms. In the long run, linear models will help us abstract, characterize, and improve interconnected networks of biometric devices. The existence of a global linear model opens up the possibility of the use of concepts of highly successful linear system theory to the analysis of interconnected biometric systems.

\section{REFERENCES}

[1] P. Wang, Q. Ji, and J. Wayman, "Modeling and predicting face recognition system performance based on analysis of similarity scores," IEEE Transactions on Pattern Analysis and Machine Intelligence, vol. 29, no. 4 , pp. 665-670, 2007.

[2] S. Mitra, M. Savvides, and A. Brockwell, "Statistical performance evaluation of biometric authentication systems using random effects models," IEEE Transactions on Pattern Analysis and Machine Intelligence, vol. 29, no. 4, pp. 517-530, 2007.

[3] R. Wang and B. Bhanu, "Learning models for predicting recognition performance," in IEEE International Conference on Computer Vision (ICCV), pp. 1613-1618, 2005.

[4] P. Grother and P. J. Phillips, "Models of large population recognition performance," in IEEE Conference on Computer Vision and Pattern Recognition, pp. 68-75, 2004.

[5] J. Han and B. Bhanu, "Performance prediction for individual recognition by gait," Pattern Recognition Letters, vol. 26, no. 5, pp. 615-624, 2005 .

[6] K. Nandakumar, Y. Chen, S. C. Dass, and A. Jain, "Likelihood ratiobased biometric score fusion," IEEE Transactions on Pattern Analysis and Machine Intelligence, vol. 30, no. 2, pp. 342-347, 2008.

[7] T. Cox and M. Cox, Multidimensional Scaling. Chapman and Hall, second ed., 1994.

[8] S. Roweis and L. Saul, "Nonlinear dimensionality reduction by locally linear embedding," Science Magazine, vol. 290, no. 5500, pp. 23232326,2000

[9] J. Tenenbaum, V. Silva, and J. Langford, "A global geometric framework for nonlinear dimensionality reduction," Science Magazine, vol. 290, no. 5500, pp. 2319-2323, 2000.

[10] A. O'Toole, C. Yi, P. J. Phillips, B. Ross, and H. A. Wild, "Face recognition algorithms as models of human face processing," in International Conference on Automatic Face and Gesture Recognition, pp. 552-557, 2000.

[11] P. Mohanty, S. Sarkar, and R. Kasturi, "From scores to face template: A model-based approach," IEEE Transactions on Pattern Analysis and Machine Intelligence, vol. 29, no. 12, pp. 2065-2078, 2007.

[12] P. Mohanty, Learning from Biometric Distances: Performance and Security Related Issues in Face Recognition Systems. PhD thesis, University of South Florida, 2007.

[13] E. Pekalska, P. Paclik, and R. Duin, "A generalized kernel approach to dissimilarity based classification," Journal of Machine Learning Research, vol. 2, pp. 175-211, 2001.

[14] V. Roth, J. Laub, M. Kawanabe, and J. M. Buhmann, "Optimal cluster preserving embedding of nonmetric proximity data," IEEE Transactions on Pattern Analysis and Machine Intelligence, vol. 25, no. 12, pp. 1540-1551, 2003.

[15] R. Reiss and M. Thomas, Statistical analysis of extreme values. Birkhauser Verlag Boston, 2001.

[16] P. J. Phillips, H. Moon, S. A. Rizvi, and P. J. Rauss, "The FERET evaluation methodology for face-recognition algorithms," IEEE Transactions on Pattern Analysis and Machine Intelligence, vol. 22, no. 10, pp. 1090-1104, 2000.

[17] S. Sarkar, P. J. Phillips, Z. Liu, I. Vega, P. Grother, and K. Bowyer, "The HumanID Gait Challenge Problem: Data Sets, Performance, and Analysis," IEEE Transactions on Pattern Analysis and Machine Intelligence, pp. 162-177, 2005.

[18] Image Group of the IAD at NIST, "Biometric scores set - release 1 (bssr1)." http://www.itl.nist.gov/iad/894.03/biometricscores/.

[19] D. Bolme, "Elastic bunch graph matching," in Masters Thesis, Colorado State University, 2003. 